\title{
Tratamento enzimático da polpa de uva no processo de produçáo de suco de uva
}

\author{
Plinio Ribeiro Fajardo Campos ${ }^{1}$ \\ Aparecido Nivaldo Módenes ${ }^{2}$ \\ Fernando Rodolfo Espinoza-Quiñones ${ }^{2}$ \\ Daniela Estelita Goes Trigueros ${ }^{2}$ \\ Sueli Teresa Davantel Barros ${ }^{1}$ \\ Nehemias Curvelo Pereira ${ }^{1}$
}

\begin{abstract}
Resumo: Este trabalho tem como objetivo investigar as melhores condiçóes para um tratamento enzimático de suco de uva. Duas enzimas do tipo pectinolyase (Novozym 33.095) e poligalacturonase (Ultrazym AFP L) foram testadas a fim de melhorar a qualidade da polpa de uva antes de um processo de separaçáo de membrana. Um conjunto de tratamentos enzimáticos foi realizado considerando uma gama de concentraçóes enzimáticas (50-100 $\left.\mathrm{mgL}^{-1}\right)$, temperaturas de tratamento $\left(35\right.$ e $\left.45^{\circ} \mathrm{C}\right)$ e tempos de processo $(0-90 \mathrm{~min}$.). Essencialmente um conjunto de parâmetros físico-químicos, tais como a acidez total, sólidos solúveis, cor e turbidez foi considerado para a atribuição de qualidade da polpa e a escolha da enzima, assim como obter a sua melhor condição experimental. Da análise estatística de Tukey dos dados para ambas as enzimas testadas, um tratamento enzimático baseado na Novozym 33095 melhorou a qualidade da polpa quando este processo foi realizado a uma concentração enzimática de $50 \mathrm{mg} \mathrm{L}^{-1}, 35^{\circ} \mathrm{C}$ e tempo de tratamento de 15 min. Sob esta condição experimental, uma redução significativa da turbidez, sólidos em suspensão e pectina na uva foi alcançada, resultando em um suco de uva com uma mínima quantidade de polpa, mais clara e transparente.
\end{abstract}

Palavras-chave: suco de uva; tratamento enzimático; parâmetros físico-químicos.

Abstract: This work aims to investigate the best conditions for an enzymatic treatment of grape juice. Two enzymes of type pectinolyase (Novozym 33095) and polygalacturonase (Ultrazym AFP L) were tested in order to improve the quality of the pulp of grape before a membrane separation process. A series of enzymatic treatments was performed regarding properly ranges of enzymatic concentrations $\left(50-100 \mathrm{mgL}^{-1}\right)$, treatment temperatures $\left(35\right.$ and $45^{\circ} \mathrm{C}$ ) and process times (0-90 min.). Essentially a set of physico-chemical parameters such as total acidity, soluble solids, color and turbidity was considered for the assignment of grape pulp quality and choose of the enzyme as well as get its best experimental condition. From a statistical analysis for both tested enzymes, an enzymatic treatment based on the Novozym 33095 has improved the grape pulp quality when this process is performed at an enzymatic concentration of $50 \mathrm{mg} \mathrm{L}^{-1}, 35^{\circ} \mathrm{C}$ temperature and $15 \mathrm{~min}$. of treatment time. Under this experimental condition, a significant reduction on the turbidity, suspended solids and pectin in grape was achieved, resulting in a grape juice with a minimum amount of pulp, more clear and transparent.

Keywords: grape juice; enzymatic treatment; physico-chemical parameters.

\footnotetext{
1 Programa de Pós-Graduação em Engenharia Química - Universidade Estadual de Maringá - Av. Colombo, 579. Campus Universitário - Maringá - PR - Brasil

2 Programa de Pós-Graduação em Engenharia Química - Universidade Estadual do Oeste do Paraná, Campus de Toledo - Rua da Faculdade, 645. Jardim Santa Maria - Toledo - PR - Brasil
} 


\section{INTRODUÇÃO}

Com vistas a atender padróes de qualidade e outras exigências do mercado consumidor referente à produção e comercializaçáo de diferentes tipos de sucos de frutas torna-se necessário a aplicação de tecnologias inovadoras. Os parâmetros físico-químicos tais como turbidez, cor, $\mathrm{pH}$, e sólidos solúveis ( ${ }^{\circ} B$ rix), são comumente utilizados como indicadores da qualidade do produto final. A turbidez nos sucos ocorre devido à presença de substâncias em suspensão neles contidos, tais como lipídios, amido, celulose, taninos e principalmente pectinas.

Visando melhorar a qualidade do produto, aplicam-se os métodos convencionais de separação, tais como a filtração, centrifugação, destilação, evaporaçáo e sedimentação. No entanto, os processos de separação por membranas (PSM) apresentam-se como uma alternativa aos métodos convencionais de separaçáo devido à alta seletividade, ao baixo consumo de energia e ao menor tempo de operação (Habert et al. 2006). Além disso, processos com membranas não requerem a utilização de produtos químicos, o que implica na minimização da geração de efluentes contaminados. Assim, entre as técnicas mais modernas para a clarificação, concentração, fracionamento, dessalinização e purificação de uma variedade de bebidas, os PSM têm um papel importante contribuindo para melhoria da segurança alimentar dos produtos finais, sem a necessidade de tratamento térmico (Marzarotto, 2005). Nas últimas décadas, os PSM em indústrias alimentícias têm sido aplicados e a sua integração com métodos convencionais tem ganho destaque na industrialização de sucos de fruta, devido à minimizaçáo de custos com matérias-prima e equipamentos e à maior qualidade do produto final. Dentre as aplicaçóes realizadas com sucesso tem-se: a osmose inversa para a concentração; a ultrafiltração para o fracionamento, concentração e purificação; a microfiltração para a clarificação, esterilização e fracionamento de macromoléculas; eletrodiálise para a desmineralização e a pervaporação para a extração e/ou destilação (Cheryan e Alvarez, 1995). Assim, os processos convencionais combinados aos PSM têm apresentado vantagens se comparado a aplicaçáo de processos de separaçáo isoladamente (Yamaguchi et al., 2007). A qua- lidade do produto clarificado por microfiltração apresenta melhora devido à maior capacidade de retenção das partículas formadoras da turbidez e principalmente pela a ausência da etapa de pasteurização (Cheryan e Alvarez, 1995), sendo esta última considerada a grande vantagem da aplicação dos PSM na clarificação e concentração de sucos de fruta (Cassano et al., 2003).

Visto que os sucos de frutas despolpados são ricos em partículas insolúveis e em materiais em suspensão, os pré-tratamentos, tais como as reaçôes químicas e enzimáticas, a centrifugação e a pré-filtração, são essenciais como etapas preliminares aos processos de micro/ultrafiltraçáo para preservar a eficiência da membrana, minimizando a formação de camada de torta e a polarização por concentração. As pesquisas desenvolvidas sobre os processos bioquímicos indicaram a utilização de enzimas de maceraçáo (pectinases, celulases e hemicelulases) durante o processo de industrialização, principalmente como prétratamento para sucos a serem clarificados por micro ou por ultrafiltração (Petrus, 1997; Balischi et al., 2002). Segundo Barros (2002), o tratamento enzimático aumenta o tamanho das partículas de sólidos suspensos, devido à redução da repulsão eletrostática entre as nuvens de partículas, fazendo-as se agruparem. Dependendo da distribuição do tamanho das partículas do produto a ser ultrafiltrado e dos poros da membrana (na micro/ultrafiltraçáo e osmose inversa), pode ocorrer um bloqueio completo ou parcial destes, com conseqüente formaçáo de uma camada secundária, que passa, então, a constituir o próprio meio filtrante. Esses mecanismos influem diretamente no fluxo de permeado da membrana. Neste contexto, o tratamento enzimático torna-se uma etapa necessária no processo de fabricação de sucos.

Assim, este trabalho tem por objetivo avaliar as melhores condiçóes para o tratamento enzimático da polpa de uva visando a reduçáo dos teores de sólidos em suspensão e de pectina. Foram testadas as enzimas pectino liase (novozym 33095) e poligalacturonase (ultrazym AFP L). Como parâmetros de controle foram considerados a acidez total, sólidos solúveis ( ${ }^{\circ}$ Brix), cor, $\mathrm{pH}$, sólidos totais e turbidez. 


\section{MATERIAIS E MÉTODOS}

\subsection{COLETA E PREPARO DA POLPA}

Devido a sua alta produção na regiáo de Toledo-Pr e apresentar melhores características físicas e químicas na produção de suco de uva, a uva Isabel foi escolhida como matéria prima. Após a coleta das uvas, estas foram despolpadas em equipamento industrial da Mercopolpa para a retirada de cascas e sementes, sendo em seguida, acondicionadas em embalagens plásticas de $15 \mathrm{~kg}$ e armazenadas à temperatura de $-4^{\circ} \mathrm{C}$.

\subsection{ENZIMAS TESTADAS}

Foram testadas dois tipos de enzimas da empresa Novozymes Latin America Ltda: a pectino liase Novozym $33095^{\circ}$ com melhor atividade pectolítica nas temperaturas de 35 e $45^{\circ} \mathrm{C}$ e a poligalacturonase celulase Ultrazym AFP $\mathrm{L}^{\oplus} \mathrm{com}$ maior porcentagem de atividade pectolítica nas temperaturas de 25 a $35^{\circ} \mathrm{C}$ (Novozymes, 2001). As concentraçóes utilizadas para as enzimas foram de 50, 100 e 150 ppm, de acordo com Balischi (2002).

\subsection{ANÁLISES FÍSICO-QUÍMICAS}

No laboratório, as polpas de uva foram descongeladas a temperatura ambiente e submetidas a análises físico químicas para determinação do $\mathrm{pH}$, Cor, Turbidez, Sólidos solúveis ( ${ }^{\circ}$ Brix) e acidez total. As medidas de $\mathrm{pH}$ dos sucos de uva foram realizadas utilizando um pHmetro Digimed DM 20. A cor tanto do suco despolpado como do clarificado foi determinada pela medida da absorbância de luz no comprimento de onda de $440 \mathrm{~nm}$, realizado em espectrofotômetro UV-vis (marca Shimadzu, modelo HACH DR/2010). A partir das leituras de absorbância, o grau de clarificação dos sucos de uva foi também determinado e expresso em porcentagem de transmitância. A turbidez dos sucos de uva foi também determinada por meio da leitura de absorbância, porém no comprimento de onda de $860 \mathrm{~nm}$. A determinação de acidez foi feita por método titulométrico, utilizando $\mathrm{NaOH}$ e fenolftaleína como indica- dor conforme descrito por Instituto Adolfo Lutz, 1985. A quantidade de sólidos solúveis ( $\left.{ }^{\circ} \mathrm{Brix}\right)$ foi determinado utilizando um refratômetro (marca Shimadzu, modelo ABBE-3L) conforme metodologia descrita por A.O.A.C (1990)

\subsection{TRATAMENTO ENZIMÁTICO}

A metodologia do tratamento enzimático consiste em adicionar $100 \mathrm{~mL}$ de polpa, previamente descongelada e homogeneizada, em um erlenmeyer de $250 \mathrm{~mL}$ sob agitação e temperatura controlada. Após atingida a temperatura desejada, adiciona-se a enzima (Balischi et al., 2002).

Baseado num delineamento experimental completamente casualizado, os tratamentos enzimáticos, em banho termostatizado com agitação controlada, foram realizados considerando duas temperaturas de 35 e $45^{\circ} \mathrm{C}$ para a enzima Novozym $33095^{\circ}$ e duas temperaturas de 25 a $35^{\circ} \mathrm{C}$ para a Ultrazym AFP $\mathrm{L}^{\oplus}$, fazendo combinaçóes com as concentraçóes de 50, 100 e 150 ppm e os tempos de tratamentos de 15 a 90 min., a intervalos de 15 minutos. Após cada tratamento enzimático, a desativação da enzima foi realizada colocando-se as amostras em banho termostatizado à $70{ }^{\circ} \mathrm{C}$ por cerca de 20 minutos. Para fins de comparação de resultados, foi utilizada uma amostra controle sem adiçáo de enzimas. No intuito de avaliar os parâmetros de resposta indicadores da qualidade do produto final após a série de tratamentos, foram realizadas as análises de acidez total, sólidos solúveis ( $\left.{ }^{\circ} \mathrm{Brix}\right)$, cor, $\mathrm{pH}$, sólidos totais e turbidez.

Com o objetivo de avaliar se os conjuntos de dados dos parâmetros de resposta (acidez total, sólidos solúveis ( ${ }^{\circ} \mathrm{Brix}$ ), cor, $\mathrm{pH}$, sólidos totais $\mathrm{e}$ turbidez) seguem a distribuição normal, a hipótese de nulidade foi testada para um nível $\mathrm{Q}=0,05$, baseado no teste Shapiro-Wilk. Confirmada a distribuição normal dos dados de resposta, os valores médios dos parâmetros físico-químicos foram avaliados quanto a sua significância segundo as condiçóes experimentais do tratamento enzimático, aplicando a ferramenta estatística ANOVA e obtendo os resultados do teste $\mathrm{F}$ para as médias. As diferenças estatisticamente significativas, em nível de 95\% ( $\mathrm{p}<0,05)$, entre as médias múltiplas dos parâmetros físico-químicos também foram avaliadas baseadas no teste Tukey. 


\section{RESULTADOS E DISCUSSÁO}

\subsection{CARACTERIZAÇÃO DA POLPA}

A polpa de uva, sem nenhum tratamento, foi caracterizada por meio de análises dos parâmetros físico-químicos: acidez total, Sólidos soluveis ( ${ }^{\circ}$ Brix), cor, $\mathrm{pH}$, sólidos totais e turbidez, cujos resultados são apresentados na Tabela 1 .

Tabela 1. Parâmetros físico-químicos da polpa original.

\begin{tabular}{|l|c|}
\hline Parâmetros & Polpa original \\
\hline acidez total titulável $(\% \mathrm{v} / \mathrm{m})$ & 13,9 \\
\hline sólidos solúveis $\left({ }^{\circ} \mathrm{Brix}\right)$ & 11,0 \\
\hline Cor $(\mathrm{mg}$ Co-Pt $/ \mathrm{L})$ & 31000 \\
\hline $\mathrm{pH}$ & 3,1 \\
\hline Sólidos Totais $(\% \mathrm{~m} / \mathrm{m})$ & 7,7 \\
\hline Turbidez $(\mathrm{FAU})$ & 8700 \\
\hline
\end{tabular}

Embora na literatura as informaçóes do padrão de qualidade do suco de uva náo são uniformes, pois depende muito da procedência da polpa de uva, o tipo de tratamento, e da obtenção de características finais vinculadas ao aroma, sabor e aparência final do suco, foram escolhidos neste trabalho os parâmetros físico-químicos: acidez total, sólidos, cor, $\mathrm{pH}$, sólidos totais e turbidez como indicadores da qualidade do produto final, sendo desejáveis que os valores dos parâmetros cor e $\mathrm{pH}$ se mantenham inalterados, enquanto que os valores de parâmetros tais como sólidos totais, turbidez e cor devam ser reduzidos, a fim de se obter um suco de uva que atenda as exigências do mercado consumidor.

\subsection{AVALIAÇÃO DOS PARÂMETROS FÍSICO-QUÍMICOS}

Os resultados obtidos do delineamento experimental executado para os parâmetros acidez total, sólidos solúveis ( $\left.{ }^{\circ} \mathrm{Brix}\right)$, cor, $\mathrm{pH}$, sólidos totais e turbidez são apresentados nas Tabelas 2 e 3 para as enzimas Novozym 33095 e Ultrazym AFP $\mathrm{L}$, respectivamente.

Devido a quantidade de dados apresentados nas Tabelas 2 e 3, para um direcionamento da melhor condição para realização do tratamento enzimático as respostas das análises físico-químicas obtidas foram submetidas a análises estatísticas. Os testes de nulidade mostraram que os dados das variáveis de resposta (acidez total, sólidos solúveis, cor, $\mathrm{pH}$, sólidos totais e turbidez) seguem uma distribuição normal. Foi observado que o teste $\mathrm{F}$ e o teste Tukey aplicado às médias das variáveis de resposta da qualidade do suco de uva mostraram resultados muito similares, por conseguinte será apresentado o resultado da análise estatística obtido pelo teste Tukey.

$\mathrm{Na}$ aplicação do teste Tukey para a escolha da enzima mais eficiente juntamente com a melhor condição experimental para o tratamento enzimático da amostra, o software SAS ${ }^{\circledR} 9.1$ fornece como primeiro resultado a menor média do conjunto de valores médios das variáveis de reposta (acidez total, sólidos solúveis, cor, $\mathrm{pH}$, sólidos totais e turbidez) quando se compara os resultados das três concentraçôes de enzima (50, 100 e 150 ppm) e do conjunto de tempos de tratamento (0 a 90 minutos a intervalos de $15 \mathrm{~min}$ ) para as duas temperaturas de tratamento enzimático $(35$ e 45 para enzima Novozym 33095 e 25 e 35 para a enzima Ultrazym AFP L). Assim, os menores valores médios do conjunto de dados para a acidez total, os sólidos solúveis, a cor, o $\mathrm{pH}$, os sólidos totais e a turbidez são apresentados na Tabela 3 para ambas as enzimas sob estudo. 
Tabela 2. Parâmetros físico-químicos da polpa de uva obtido após o tratamento utilizando a enzima Novozym 33095.

\begin{tabular}{|c|c|c|c|c|c|c|c|c|c|}
\hline \multirow{2}{*}{ Parâmetro } & \multirow{2}{*}{$\begin{array}{l}\text { Tempera- } \\
\text { tura }\left({ }^{\circ} \mathrm{C}\right)\end{array}$} & \multirow{2}{*}{$\begin{array}{l}\text { Concentra- } \\
\text { ção }\left(\mathrm{mgL}^{-1}\right)\end{array}$} & \multicolumn{7}{|c|}{ Tempo (mim) } \\
\hline & & & 0 & 15 & 30 & 45 & 60 & 75 & 90 \\
\hline \multirow{6}{*}{$\begin{array}{l}\text { Acidez } \\
(\% \mathrm{v} / \mathrm{m})\end{array}$} & \multirow{3}{*}{35} & 50 & $13,9 \pm 0,7$ & $12,8 \pm 0,6$ & $12,3 \pm 0,6$ & $12,3 \pm 0,6$ & $12,3 \pm 0,6$ & $12,8 \pm 0,6$ & $13,4 \pm 0,7$ \\
\hline & & 100 & $13,90,7$ & $12,8 \pm 0,6$ & $14,5 \pm 0,7$ & $12,3 \pm 0,6$ & $12,3 \pm 0,6$ & $12,8 \pm 0,6$ & $12,8 \pm 0,6$ \\
\hline & & 150 & $13,9 \pm 0,7$ & $12,8 \pm 0,6$ & $16,2 \pm 0,8$ & $15,1 \pm 0,8$ & $15,6 \pm 0,8$ & $17,3 \pm 0,9$ & $15,6 \pm 0,8$ \\
\hline & \multirow{3}{*}{45} & 50 & $14,50,7$ & $13,9 \pm 0,7$ & $13,4 \pm 0,7$ & $12,8 \pm 0,6$ & $13,4 \pm 0,7$ & $13,4 \pm 0,7$ & $13,9 \pm 0,7$ \\
\hline & & 100 & $13,90,7$ & $12,8 \pm 0,6$ & $13,4 \pm 0,7$ & $15,1 \pm 0,8$ & $16,2 \pm 0,8$ & $13,9 \pm 0,7$ & $14,5 \pm 0,7$ \\
\hline & & 150 & $18,4 \pm 0,9$ & $16,7 \pm 0,8$ & $17,3 \pm 0,9$ & $16,7 \pm 0,8$ & $17,3 \pm 0,9$ & $16,7 \pm 0,8$ & $17,3 \pm 0,9$ \\
\hline \multirow{6}{*}{$\begin{array}{l}\text { Sólidos } \\
\text { solúveis } \\
\text { ('Brix) }\end{array}$} & \multirow{3}{*}{35} & 50 & $7,9 \pm 0,4$ & $7,4 \pm 0,4$ & $7,0 \pm 0,4$ & $7,0 \pm 0,4$ & $7,0 \pm 0,4$ & $7,8 \pm 0,4$ & $7,2 \pm 0,4$ \\
\hline & & 100 & $8,00,4$ & $6,8 \pm 0,3$ & $7,0 \pm 0,4$ & $7,4 \pm 0,4$ & $7,4 \pm 0,4$ & $7,8 \pm 0,4$ & $7,4 \pm 0,3$ \\
\hline & & 150 & $10,0 \pm 0,5$ & $9,8 \pm 0,5$ & $9,8 \pm 0,5$ & $9,8 \pm 0,5$ & $9,8 \pm 0,5$ & $10,8 \pm 0,5$ & $9,8 \pm 0,5$ \\
\hline & \multirow{3}{*}{45} & 50 & $13,50,7$ & $11,0 \pm 0,6$ & $13,9 \pm 0,7$ & $10,9 \pm 0,6$ & $10,9 \pm 0,6$ & $13,1 \pm 0,7$ & $11,9 \pm 0,6$ \\
\hline & & 100 & $10,20,5$ & $12,9 \pm 0,6$ & $13,9 \pm 0,7$ & $14,5 \pm 0,7$ & $13,8 \pm 0,7$ & $12,1 \pm 0,6$ & $11,1 \pm 0,6$ \\
\hline & & 150 & $13,1 \pm 0,7$ & $12,0 \pm 0,6$ & $11,2 \pm 0,6$ & $11,2 \pm 0,6$ & $11,8 \pm 0,6$ & $11,8 \pm 0,6$ & $11,2 \pm 0,6$ \\
\hline \multirow{6}{*}{$\begin{array}{c}\text { Cor } \\
(1.000 \mathrm{mg} \\
\text { PCto } / \mathrm{L})\end{array}$} & \multirow{3}{*}{35} & 50 & $31,5 \pm 0,9$ & $25,9 \pm 0,8$ & $25,0 \pm 0,8$ & $28,7 \pm 0,9$ & $26,8 \pm 0,8$ & $34,3 \pm 1$ & $21,2 \pm 0,6$ \\
\hline & & 100 & $31,50,9$ & $22,3 \pm 0,7$ & $23,8 \pm 0,7$ & $25,0 \pm 0,8$ & $26,5 \pm 0,8$ & $26,5 \pm 0,8$ & $28,6 \pm 0,9$ \\
\hline & & 150 & $14,3 \pm 0,4$ & $25,4 \pm 0,8$ & $35,6 \pm 1,1$ & $38,6 \pm 1,2$ & $32,3 \pm 1,2$ & $26,5 \pm 0,8$ & $22,6 \pm 0,7$ \\
\hline & \multirow{3}{*}{45} & 50 & 34,01 & $22,3 \pm 0,7$ & $25,6 \pm 0,8$ & $27,0 \pm 0,8$ & $29,7 \pm 0,9$ & $36,5 \pm 1,1$ & $28,6 \pm 0,9$ \\
\hline & & 100 & $8,20,8$ & $29,2 \pm 0,9$ & $43,7 \pm 1,3$ & $26,8 \pm 0,8$ & $38,7 \pm 1,2$ & $36,3 \pm 1,1$ & $35,7 \pm 1,1$ \\
\hline & & 150 & $20,9 \pm 0,6$ & $20,1 \pm 0,6$ & $26,0 \pm 0,8$ & $23,1 \pm 0,7$ & $19,8 \pm 0,6$ & $21,3 \pm 0,6$ & $29,4 \pm 0,9$ \\
\hline \multirow{6}{*}{$\mathrm{pH}$} & \multirow{3}{*}{35} & 50 & $3,360,02$ & $3,40 \pm 0,02$ & $3,40 \pm 0,023$ & $3,28 \pm 0,02$ & $3,28 \pm 0,02$ & $3,35 \pm 0,02$ & $3,34 \pm 0,02$ \\
\hline & & 100 & $3,260,02$ & $3,23 \pm 0,02$ & $3,23 \pm 0,023$ & $3,28 \pm 0,05$ & $3,32 \pm 0,02$ & 3,21 & $3,31 \pm 0,02$ \\
\hline & & 150 & $3,16 \pm 0,02$ & $3,20 \pm 0,02$ & $3,20 \pm 0,013$ & $3,18 \pm 0,02$ & $3,12 \pm 0,0$ & 3,20 & $3,20 \pm 0,02$ \\
\hline & \multirow{3}{*}{45} & 50 & $3,320,02$ & $3,29 \pm 0,02$ & $3,26 \pm 0,023$ & $3,19 \pm 0,02$ & $3,18 \pm 0,02$ & $3,15 \pm 0,02$ & $3,08 \pm 0,02$ \\
\hline & & 100 & $3,220,02$ & $3,23 \pm 0,02$ & $3,23 \pm 0,023$ & $3,23 \pm 0,02$ & $3,22 \pm 0,02$ & $3,27 \pm 0,02$ & $3,21 \pm 0,02$ \\
\hline & & 150 & $3,09 \pm 0,02$ & $3,10 \pm 0,02$ & $3,15 \pm 0,02$ & $3,16 \pm 0$ & $3,07 \pm 0,02$ & $3,10 \pm 0,02$ & $3,15 \pm 0,02$ \\
\hline \multirow{6}{*}{$\begin{array}{c}\text { Sólidos } \\
\text { Totais } \\
(\% \mathrm{~m} / \mathrm{m})\end{array}$} & \multirow{3}{*}{35} & 50 & $7,7 \pm 0,4$ & $6,7 \pm 0,3$ & $6,9 \pm 0,3$ & $6,9 \pm 0,3$ & $7,0 \pm 0,4$ & $7,0 \pm 0,4$ & $7,0 \pm 0,4$ \\
\hline & & 100 & $7,40,4$ & $7,4 \pm 0,4$ & $7,4 \pm 0,4$ & $6,6 \pm 0,3$ & $6,8 \pm 0,3$ & $6,8 \pm 0,3$ & $6,7 \pm 0,3$ \\
\hline & & 150 & $8,3 \pm 0,4$ & $8,2 \pm 0,4$ & $8,5 \pm 0,4$ & $8,7 \pm 0,4$ & $8,2 \pm 0,4$ & $8,8 \pm 0,4$ & $9,1 \pm 0,5$ \\
\hline & \multirow{3}{*}{45} & 50 & $8,00,4$ & $7,4 \pm 0,4$ & $7,4 \pm 0,4$ & $7,3 \pm 0,4$ & $7,4 \pm 0,4$ & $6,9 \pm 0,3$ & $7,7 \pm 0,4$ \\
\hline & & 100 & $7,90,4$ & $7,4 \pm 0,4$ & $8,9 \pm 0,4$ & $8,6 \pm 0,4$ & $8,9 \pm 0,4$ & $9,0 \pm 0,5$ & $8,5 \pm 0,4$ \\
\hline & & 150 & $11,1 \pm 0,6$ & $9,5 \pm 0,5$ & $9,6 \pm 0,5$ & $9,4 \pm 0,5$ & $9,4 \pm 0,5$ & $9,4 \pm 0,5$ & $9,2 \pm 0,5$ \\
\hline \multirow{6}{*}{$\begin{array}{c}\text { Turbidez } \\
(1.000 \\
\text { FAU })\end{array}$} & \multirow{3}{*}{35} & 50 & $8,7 \pm 0,3$ & $4,5 \pm 0,1$ & $4,5 \pm 0,1$ & $9,5 \pm 0,3$ & $6,9 \pm 0,2$ & $5,1 \pm 0,2$ & $6,5 \pm 0,2$ \\
\hline & & 100 & $7,10,2$ & $5,3 \pm 0,1$ & $9,1 \pm 0,3$ & $9,1 \pm 0,3$ & $8,0 \pm 0,2$ & $6,3 \pm 0,2$ & $5,9 \pm 0,2$ \\
\hline & & 150 & $3,00,1$ & $7,8 \pm 0,2$ & $7,8 \pm 0,2$ & $13,2 \pm 0,4$ & $10,2 \pm 0,3$ & $11,4 \pm 0,3$ & $3,7 \pm 0,1$ \\
\hline & \multirow{3}{*}{45} & 50 & $9,10,3$ & $6,5 \pm 0,2$ & $7,3 \pm 0,2$ & $8,3 \pm 0,3$ & $7,3 \pm 0,2$ & $9,3 \pm 0,3$ & $10,9 \pm 0,3$ \\
\hline & & 100 & $5,70,2$ & $5,5 \pm 0,2$ & $6,3 \pm 0,2$ & $11,4 \pm 0,3$ & $11,1 \pm 0,3$ & $12,3 \pm 0,4$ & $12,5 \pm 0,4$ \\
\hline & & 150 & $5,3 \pm 0,2$ & $4,2 \pm 0,1$ & $6,3 \pm 0,2$ & $5,0 \pm 0,2$ & $4,0 \pm 0,1$ & $6,1 \pm 0,2$ & $7,5 \pm 0,2$ \\
\hline
\end{tabular}


$\mathrm{Na}$ aplicação do teste Tukey para a escolha da enzima mais eficiente juntamente com a melhor condiçáo experimental para o tratamento enzimático da amostra, o software SAS ${ }^{\circledR} 9.1$ fornece como primeiro resultado a menor média do conjunto de valores médios das variáveis de reposta (acidez total, sólidos solúveis, cor, $\mathrm{pH}$, sólidos totais e turbidez) quando se compara os resultados das três concentraçóes de enzima (50, 100 e 150 ppm) e do conjunto de tempos de tratamento (0 a 90 minutos a intervalos de $15 \mathrm{~min}$ ) para as duas temperaturas de tratamento enzimático $(35$ e 45 para enzima Novozym 33095 e 25 e 35 para a enzima Ultrazym AFP L). Assim, os menores valores médios do conjunto de dados para a acidez total, os sólidos solúveis, a cor, o $\mathrm{pH}$, os sólidos totais e a turbidez são apresentados na Tabela 3 para ambas as enzimas sob estudo.

Tabela 3. Parâmetros físico-químicos da polpa de uva obtido após o tratamento utilizando a enzima Ultrazym AFP L

\begin{tabular}{|c|c|c|c|c|c|c|c|c|c|}
\hline \multirow{2}{*}{$\begin{array}{l}\text { Parâme- } \\
\text { tro }\end{array}$} & \multirow{2}{*}{$\begin{array}{c}\text { Tempe- } \\
\text { ratura } \\
\left({ }^{\circ} \mathrm{C}\right)\end{array}$} & \multirow{2}{*}{$\begin{array}{c}\text { Concen- } \\
\text { tração } \\
\left(\mathrm{mgL}^{-1}\right)\end{array}$} & \multicolumn{7}{|c|}{ Tempo (mim) } \\
\hline & & & 0 & 15 & 30 & 45 & 60 & 75 & 90 \\
\hline \multirow{6}{*}{$\begin{array}{c}\text { Acidez } \\
(\% \mathrm{v} / \mathrm{m})\end{array}$} & \multirow{3}{*}{25} & 50 & $18,9 \pm 0,8$ & $17,3 \pm 0,8$ & $17,3 \pm 0,8$ & $17,3 \pm 0,8$ & $16,7 \pm 0,8$ & $16,2 \pm 0,8$ & $15,1 \pm 0,8$ \\
\hline & & 100 & $13,9 \pm 0,7$ & $14,5 \pm 0,7$ & $15,60,7$ & $17,3 \pm 0,8$ & $16,7 \pm 0,8$ & $17,3 \pm 0,7$ & $17,9 \pm 0,8$ \\
\hline & & 150 & $16,7 \pm 0,8$ & $15,6 \pm 0,7$ & $15,10,7$ & $14,5 \pm 0,6$ & $14,5 \pm 0,6$ & $14,5 \pm 0,6$ & $15,6 \pm 0,7$ \\
\hline & \multirow{3}{*}{35} & 50 & $18,9 \pm 0,9$ & $17,9 \pm 0,9$ & $18,40,9$ & $17,3 \pm 0,9$ & $16,7 \pm 0,8$ & $18,4 \pm 0,9$ & $16,2 \pm 0,8$ \\
\hline & & 100 & $18,9 \pm 0,9$ & $17,9 \pm 0,9$ & $18,40,9$ & $17,9 \pm 0,9$ & $17,9 \pm 0,9$ & $17,9 \pm 0,9$ & $18,4 \pm 0,9$ \\
\hline & & 150 & $17,3 \pm 0,8$ & $16,7 \pm 0,8$ & $17,90,9$ & $17,9 \pm 0,9$ & $16,2 \pm 0,8$ & $12,8 \pm 0,6$ & $13,4 \pm 0,7$ \\
\hline \multirow{6}{*}{$\begin{array}{c}\text { Sólidos } \\
\text { solúveis } \\
\left({ }^{\circ} \mathrm{Brix}\right)\end{array}$} & \multirow{3}{*}{25} & 50 & $12,1 \pm 0,6$ & $11,1 \pm 0,6$ & $11,1 \pm 0,6$ & $11,0 \pm 0,6$ & $11,1 \pm 0,6$ & $10,2 \pm 0,5$ & $9,7 \pm 0,5$ \\
\hline & & 100 & $9,2 \pm 0,5$ & $9,2 \pm 0,5$ & $9,9 \pm 0,5$ & $10,2 \pm 0,5$ & $10,9 \pm 0,5$ & $11,0 \pm 0,6$ & $11,0 \pm 0,6$ \\
\hline & & 150 & $9,6 \pm 0,5$ & $10,2 \pm 0,5$ & $10,20,5$ & $10,0 \pm 0,5$ & $10,6 \pm 0,5$ & $10,4 \pm 0,5$ & $10,4 \pm 0,5$ \\
\hline & \multirow{3}{*}{35} & 50 & $10,8 \pm 0,5$ & $10,0 \pm 0,5$ & $11,40,6$ & $10,7 \pm 0,5$ & $10,4 \pm 0,5$ & $10,8 \pm 0,5$ & $10,4 \pm 0,5$ \\
\hline & & 100 & $12,8 \pm 0,6$ & $11,9 \pm 0,6$ & $11,80,6$ & $11,8 \pm 0,6$ & $12,0 \pm 0,6$ & $12,0 \pm 0,6$ & $12,1 \pm 0,6$ \\
\hline & & 150 & $13,0 \pm 0,7$ & $12,0 \pm 0,6$ & $12,00,6$ & $12,2 \pm 0,6$ & $9,4 \pm 0,5$ & $7,8 \pm 0,4$ & $9,2 \pm 0,4$ \\
\hline \multirow{6}{*}{$\begin{array}{c}\text { Cor } \\
(1.000 \\
\mathrm{mg} \\
\text { PCto/L) }\end{array}$} & \multirow{3}{*}{25} & 50 & $33,5 \pm 1$ & $33,3 \pm 1$ & $37,0 \pm 1,1$ & $38,9 \pm 1,2$ & $35,7 \pm 1,1$ & $42,1 \pm 1,3$ & $31,1 \pm 0,9$ \\
\hline & & 100 & $30,9 \pm 0,9$ & $33,8 \pm 1$ & 33,01 & $39,3 \pm 1,2$ & $32,2 \pm 1$ & $37,4 \pm 1,1$ & $39,2 \pm 1,2$ \\
\hline & & 150 & $45,8 \pm 1,4$ & $37,6 \pm 1,3$ & $35,51,1$ & $33,5 \pm 1$ & $34,7 \pm 1$ & $32,2 \pm 1$ & $31,2 \pm 0,9$ \\
\hline & \multirow{3}{*}{35} & 50 & $36,8 \pm 1,1$ & $40,6 \pm 1,2$ & $58,21,7$ & $48,8 \pm 1,5$ & $40,4 \pm 1,2$ & $50,8 \pm 1,5$ & $40,2 \pm 1,2$ \\
\hline & & 100 & $31,7 \pm 1$ & $39,4 \pm 1,2$ & $39,2 \pm 1,2$ & $29,3 \pm 0,9$ & $26,6 \pm 0,8$ & $26,6 \pm 0,8$ & $30,3 \pm 0,9$ \\
\hline & & 150 & $16,2 \pm 0,5$ & $33,2 \pm 1$ & $43,11,3$ & $44,1 \pm 1,3$ & $39,6 \pm 1,2$ & $38,5 \pm 1,2$ & $31,3 \pm 0,9$ \\
\hline \multirow{6}{*}{$\mathrm{pH}$} & \multirow{3}{*}{25} & 50 & $3,08 \pm 0,02$ & $3,21 \pm 0,02$ & $3,16 \pm 0,02$ & $3,16 \pm 0,02$ & $3,19 \pm 0,02$ & $3,17 \pm 0,02$ & $3,16 \pm 0,02$ \\
\hline & & 100 & $3,10 \pm 0,02$ & $3,20 \pm 0,02$ & $3,16 \pm 0,02$ & $3,15 \pm 0,02$ & $3,05 \pm 0,02$ & $3,00 \pm 0,02$ & $2,91 \pm 0,02$ \\
\hline & & 150 & $3,08 \pm 0,02$ & $3,07 \pm 0,02$ & $3,15 \pm 0,02$ & $3,11 \pm 0,02$ & $3,09 \pm 0,02$ & $2,98 \pm 0,02$ & $\pm 0,02$ \\
\hline & \multirow{3}{*}{35} & 50 & $3,12 \pm 0,02$ & $3,16 \pm 0,02$ & $3,13 \pm 0,02$ & $3,12 \pm 0,02$ & $3,10 \pm 0,02$ & $3,04 \pm 0,02$ & $2,98 \pm 0,02$ \\
\hline & & 100 & $3,09 \pm 0,02$ & $3,08 \pm 0,02$ & $3,07 \pm 0,02$ & $3,05 \pm 0,02$ & $3,06 \pm 0,02$ & $3,00 \pm 0,02$ & $2,95 \pm 0,02$ \\
\hline & & 150 & $3,12 \pm 0,02$ & $3,11 \pm 0,02$ & $3,08 \pm 0,02$ & $3,09 \pm 0,02$ & $3,08 \pm 0,02$ & $3,08 \pm 0,02$ & $3,08 \pm 0,02$ \\
\hline \multirow{6}{*}{$\begin{array}{c}\text { Sólidos } \\
\text { Totais } \\
(\% \mathrm{~m} / \mathrm{m})\end{array}$} & \multirow{3}{*}{25} & 50 & $10,0 \pm 0,5$ & $8,9 \pm 0,4$ & $8,9 \pm 0,4$ & $9,1 \pm 0,5$ & \begin{tabular}{|l|}
$8,2 \pm 0,4$ \\
\end{tabular} & $8,0 \pm 0,4$ & $8,0 \pm 0,4$ \\
\hline & & 100 & $7,9 \pm 0,4$ & $6,6 \pm 0,3$ & $8,0 \pm 0,4$ & $8,7 \pm 0,4$ & $9,2 \pm 0,5$ & $9,4 \pm 0,5$ & $9,2 \pm 0,5$ \\
\hline & & 150 & $10,3 \pm 0,5$ & $9,7 \pm 0,5$ & $9,60,5$ & $9,4 \pm 0,5$ & $8,9 \pm 0,4$ & $9,3 \pm 0,5$ & $9,3 \pm 0,5$ \\
\hline & \multirow{3}{*}{35} & 50 & $10,9 \pm 0,5$ & $9,4 \pm 0,5$ & $11,4 \pm 0,6$ & $10,2 \pm 0,5$ & $10,2 \pm 0,5$ & $10,6 \pm 0,5$ & $10,3 \pm 0,5$ \\
\hline & & 100 & $10,7 \pm 0,5$ & $10,4 \pm 0,5$ & $10,50,5$ & $11,0 \pm 0,6$ & $10,6 \pm 0,5$ & $10,8 \pm 0,5$ & $11,7 \pm 0,6$ \\
\hline & & 150 & $11,1 \pm 0,6$ & $11,3 \pm 0,6$ & $11,40,6$ & $8,9 \pm 0,4$ & $7,2 \pm 0,4$ & $7,7 \pm 0,4$ & $11,5 \pm 0,6$ \\
\hline \multirow{6}{*}{$\begin{array}{c}\text { Turbidez } \\
(1.000 \\
\text { FAU })\end{array}$} & \multirow{3}{*}{25} & 50 & $7,6 \pm 0,2$ & $7,7 \pm 0,2$ & $8,0 \pm 0,2$ & $8,8 \pm 0,3$ & $9.1 \pm 0,3$ & $14,2 \pm 0,4$ & $9,0 \pm 0,3$ \\
\hline & & 100 & $9,5 \pm 0,3$ & $10,4 \pm 0,3$ & $7,80,2$ & $10,0 \pm 0,3$ & $7,4 \pm 0,2$ & $12,1 \pm 0,4$ & $8,7 \pm 0,3$ \\
\hline & & 150 & $7,9 \pm 0,2$ & $14,0 \pm 0,4$ & $12,30,4$ & $8,1 \pm 0,2$ & $5,0 \pm 0,2$ & $5,4 \pm 0,2$ & $3,1 \pm 0,1$ \\
\hline & \multirow{3}{*}{35} & 50 & $12,8 \pm 0,4$ & $12,5 \pm 0,4$ & $12,90,4$ & $12,6 \pm 0,4$ & $8,8 \pm 0,3$ & $10,5 \pm 0,3$ & $9,5 \pm 0,3$ \\
\hline & & 100 & $7,1 \pm 0,2$ & $6,5 \pm 0,2$ & $9,3 \pm 0,3$ & $5,3 \pm 0,2$ & $6,5 \pm 0,2$ & $6,5 \pm 0,2$ & $7,1 \pm 0,2$ \\
\hline & & 150 & $4,6 \pm 0,1$ & $9,1 \pm 0,3$ & $8,8 \pm 0,3$ & $7,2 \pm 0,2$ & $9,6 \pm 0,3$ & $9,8 \pm 0,3$ & $8,2 \pm 0,2$ \\
\hline
\end{tabular}


Tabela 4. Melhor temperatura de processo utilizando as enzimas Novozym 33095 e Ultrazym AFP L de acordo com os valores médios mínimos de cada variável de reposta.

\begin{tabular}{|c|c|c|c|c|c|c|c|}
\hline \multirow{2}{*}{ Enzima } & \multirow{2}{*}{ Parâmetro } & \multicolumn{6}{|c|}{ Variáveis de resposta } \\
\cline { 3 - 8 } & $\begin{array}{c}\text { Acidez } \\
\% \mathrm{v} / \mathrm{m})\end{array}$ & $\begin{array}{c}\text { Sólidos solúveis } \\
\left({ }^{\circ} \text { Brix }\right)\end{array}$ & $\begin{array}{c}\text { Cor }(1.000 \\
\mathrm{mg} \text { PCto/L) }\end{array}$ & $\mathrm{pH}$ & $\begin{array}{c}\text { Sólidos Totais } \\
(\% \mathrm{~m} / \mathrm{m})\end{array}$ & $\begin{array}{c}\text { Turbidez } \\
(1.000 \mathrm{FAU})\end{array}$ \\
\hline \multirow{2}{*}{$\begin{array}{c}\text { Novozym } \\
33095\end{array}$} & Média mín. & 13,84 & 8,23 & 27,26 & 3,18 & 7,53 & 7,29 \\
\cline { 2 - 8 } & Temp. $\left({ }^{\circ} \mathrm{C}\right)$ & 35 & 35 & 35 & 45 & 35 & 35 \\
\hline $\begin{array}{c}\text { Ultrazym } \\
\text { AFP L }\end{array}$ & Valor mín. & 16,13 & 14,45 & 35,57 & 3,07 & 8,89 & 8,75 \\
\cline { 2 - 8 } & Temp. $\left({ }^{\circ} \mathrm{C}\right)$ & 25 & 25 & 25 & 35 & 25 & 35 \\
\hline
\end{tabular}

Como critério de análise estatístico introduzido na entrada de dados do software $\mathrm{SAS}^{\oplus}$ 9.1, foi feita inicialmente a comparação, pelo teste Tukey, dos valores médios das variáveis de resposta (acidez, sólidos solúveis, cor, $\mathrm{pH}$, sólidos totais e turbidez) para as duas temperaturas e as três concentrações consideradas para cada enzima, fixando para cada variável analisada o tempo de tratamento (Tabela 5). Em seguida, foi realizada a comparação dos valores médios das variáveis de resposta para o conjunto de tempos de tratamento e as duas temperaturas consideradas para cada enzima, fixando para cada variável analisada a concentração de cada enzima (Tabela 6)

Avaliando o parâmetro acidez, conforme o teste de Tukey, para as duas enzimas testadas, os valores obtidos relativos à variável tempo não foram significativamente diferentes $(\mathrm{p}<5 \%)$ enquanto que para a variável temperatura apresen- taram um nível de significância maior que 5\%. Foi observado um nível de significância maior que $5 \%$ para a variável concentração apenas para a enzima Ultrazym AFP L. Apesar da pequena diferença obtida nos resultados, o menor valor obtido foi para enzima Novozym 33095 nas condiçóes: temperatura de $35^{\circ} \mathrm{C}$, tempo de $15 \mathrm{~min}$. e concentração de 50 ppm.

Analisando os resultados obtidos para o parâmetro sólidos solúveis ( $\left.{ }^{\circ} \mathrm{Brix}\right)$, verifica-se que para as duas enzimas testadas, pelo teste de Tukey, os valores obtidos relativos à variável tempo não foram significativamente diferentes $(\mathrm{p}<5 \%)$ enquanto que as variáveis temperatura e concentração apresentaram um nível de significância maior que $5 \%$. Os menores valores obtidos foram para enzima Novozym 33095 nas condiçôes: temperatura de $35^{\circ} \mathrm{C}$, tempo de 15 min. e concentraçáo de $50 \mathrm{ppm}$.

Tabela 5. Resultados do teste de Tukey para os parâmetros físico-químicos para a variável tempo para as enzimas Novozym 33095 e Ultrazym AFP L.

\begin{tabular}{|c|c|c|c|c|c|c|c|c|}
\hline \multirow{2}{*}{ Parâmetro } & \multirow{2}{*}{ Enzima } & \multicolumn{7}{|c|}{ Tempo (mim) } \\
\cline { 3 - 7 } & & 0 & 15 & 30 & 45 & 60 & 75 & 90 \\
\hline \multirow{2}{*}{ Acidez $(\% \mathrm{v} / \mathrm{m})$} & Novozym 33095 & $14,79^{\mathrm{a}}$ & $14,03^{\mathrm{a}}$ & $14,51^{\mathrm{a}}$ & $14,13^{\mathrm{a}}$ & $14,51^{\mathrm{a}}$ & $14,51^{\mathrm{a}}$ & $14,59^{\mathrm{a}}$ \\
\cline { 2 - 7 } & Ultrazym AFP L & $17,49^{\mathrm{a}}$ & $16,65^{\mathrm{a}}$ & $17,11^{\mathrm{a}}$ & $17,02^{\mathrm{a}}$ & $16,45^{\mathrm{a}}$ & $16,18^{\mathrm{a}}$ & $16,08^{\mathrm{a}}$ \\
\hline \multirow{2}{*}{ Sólidos solúveis ( ${ }^{\circ}$ Brix) $)$} & Novozym 33095 & $10,45^{\mathrm{a}}$ & $9,97^{\mathrm{a}}$ & $10,47^{\mathrm{a}}$ & $10,13^{\mathrm{a}}$ & $10,12^{\mathrm{a}}$ & $10,55^{\mathrm{a}}$ & $9,98^{\mathrm{a}}$ \\
\cline { 2 - 8 } & Ultrazym AFP L & $11,25^{\mathrm{a}}$ & $10,78^{\mathrm{a}}$ & $11,10^{\mathrm{a}}$ & $11,00^{\mathrm{a}}$ & $10,75^{\mathrm{a}}$ & $10,37^{\mathrm{a}}$ & $10,42^{\mathrm{a}}$ \\
\hline \multirow{2}{*}{ Cor (mg PCto/L) } & Novozym 33095 & $26.767^{\mathrm{a}}$ & $24.167^{\mathrm{a}}$ & $29.917^{\mathrm{a}}$ & $28.200^{\mathrm{a}}$ & $28.933^{\mathrm{a}}$ & $30.177^{\mathrm{a}}$ & $27.683^{\mathrm{a}}$ \\
\cline { 2 - 8 } & Ultrazym AFP L & $32.475^{\mathrm{b}}$ & $36.233^{\mathrm{a}}$ & $40.950^{\mathrm{a}}$ & $38.950^{\mathrm{a}}$ & $34.783^{\mathrm{b}}$ & $37.850^{\mathrm{a}}$ & $33.767^{\mathrm{b}}$ \\
\hline \multirow{2}{*}{$\mathrm{pH}$} & Novozym 33095 & $3,23^{\mathrm{a}}$ & $3,24^{\mathrm{a}}$ & $3,26^{\mathrm{a}}$ & $3,21^{\mathrm{a}}$ & $3,21^{\mathrm{a}}$ & $3,21^{\mathrm{a}}$ & $3,22^{\mathrm{a}}$ \\
\cline { 2 - 8 } & Ultrazym AFP L & $3,10^{\mathrm{a}}$ & $3,14^{\mathrm{a}}$ & $3,13^{\mathrm{a}}$ & $3,11^{\mathrm{a}}$ & $3,09^{\mathrm{a}}$ & $3,05^{\mathrm{a}}$ & $2,99^{\mathrm{a}}$ \\
\hline \multirow{2}{*}{ Sólidos Totais (\%m/m) } & Novozym 33095 & $8,40^{\mathrm{a}}$ & $7,77^{\mathrm{a}}$ & $8,12^{\mathrm{a}}$ & $7,92^{\mathrm{a}}$ & $7,95^{\mathrm{a}}$ & $7,98^{\mathrm{a}}$ & $8,03^{\mathrm{a}}$ \\
\cline { 2 - 8 } & Ultrazym AFP L & $10,15^{\mathrm{a}}$ & $9,38^{\mathrm{a}}$ & $9,97^{\mathrm{a}}$ & $9,55^{\mathrm{a}}$ & $9,05^{\mathrm{a}}$ & $9,30^{\mathrm{a}}$ & $10,00^{\mathrm{a}}$ \\
\hline \multirow{2}{*}{ Turbidez (FAU) } & Novozym 33095 & $6.500^{\mathrm{b}}$ & $5.617^{\mathrm{b}}$ & $6.867^{\mathrm{b}}$ & $9.367^{\mathrm{a}}$ & $7.850^{\mathrm{a}}$ & $8.337^{\mathrm{a}}$ & $7.817^{\mathrm{a}}$ \\
\cline { 2 - 8 } & Ultrazym AFP L & $8.242^{\mathrm{a}}$ & $9.983^{\mathrm{a}}$ & $9.767^{\mathrm{a}}$ & $8.600^{\mathrm{a}}$ & $7.667^{\mathrm{a}}$ & $9.650^{\mathrm{a}}$ & $7.517^{\mathrm{a}}$ \\
\hline
\end{tabular}

(a) médias seguidas de mesma letra minúscula (a,b,c,d), dentro de cada linha, não diferem entre si a $\mathrm{p} \leq 0,05$ 
Avaliando o parâmetro cor, conforme o teste de Tukey, para a enzima Ultrazym AFP L foi observado um nível de significância maior que $5 \%$ para as variáveis concentração e tempo. Para a enzima Novozym 33095 os valores obtidos relativos às variáveis temperatura, cor e tempo não foram significativamente diferentes $(\mathrm{p}<5 \%)$.
Considerando que os valores obtidos pela enzima Novozym 33095 para a variável cor foram menores que os obtidos pela enzima Ultrazym AFP L foi escolhido como melhores condiçóes de processo o tratamento com a enzima Novozym 33095 nas condiçóes $35^{\circ} \mathrm{C}, 15 \mathrm{~min}$. e $50 \mathrm{ppm}$.

Tabela 6. Resultados do teste de Tukey para os parâmetros físico-químicos para a variável concentração para as enzimas Novozym 33095 e Ultrazym AFP L.

\begin{tabular}{|c|c|c|c|c|c|}
\hline \multirow{2}{*}{ Parâmetro } & \multirow{2}{*}{ Enzima } & \multicolumn{4}{|c|}{ Concentração (ppm) } \\
\cline { 3 - 6 } & & 0 & 50 & 100 & 150 \\
\hline \multirow{2}{*}{ Acidez (\% v/m) } & Novozym 33095 & $14,79^{\mathrm{b}}$ & $13,06^{\mathrm{c}}$ & $13,66^{\mathrm{c}}$ & $14,79^{\mathrm{b}}$ \\
\cline { 2 - 6 } & Ultrazym AFP L & $17,49^{\mathrm{a}}$ & $17,06^{\mathrm{a}}$ & $17,29^{\mathrm{a}}$ & $15,39^{\mathrm{b}}$ \\
\hline \multirow{2}{*}{ Sólidos soluveis (ํㅏix) } & Novozym 33095 & $10,45^{\mathrm{a}}$ & $9,59^{\mathrm{b}}$ & $10,18^{\mathrm{a}}$ & $10,74^{\mathrm{a}}$ \\
\cline { 2 - 6 } & Ultrazym AFP L & $11,25^{\mathrm{a}}$ & $10,69^{\mathrm{b}}$ & $11,16^{\mathrm{a}}$ & $10,36^{\mathrm{b}}$ \\
\hline \multirow{2}{*}{ Cor (mg PCto/L) } & Novozym 33095 & $26.767^{\mathrm{a}}$ & $27.600^{\mathrm{a}}$ & $30.250^{\mathrm{a}}$ & $26.683^{\mathrm{a}}$ \\
\cline { 2 - 6 } & Ultrazym AFP L & $32.475^{\mathrm{b}}$ & $41.358^{\mathrm{a}}$ & $33.792^{\mathrm{b}}$ & $36.117^{\mathrm{b}}$ \\
\hline \multirow{2}{*}{ Sólidos Totais (\%m/m) } & Novozym 33095 & $3,23^{\mathrm{a}}$ & $3,27^{\mathrm{a}}$ & $3,25^{\mathrm{a}}$ & $3,16^{\mathrm{a}}$ \\
\cline { 2 - 6 } & Ultrazym AFP L & $3,10^{\mathrm{a}}$ & $3,13^{\mathrm{a}}$ & $3,06^{\mathrm{a}}$ & $3,06^{\mathrm{a}}$ \\
\hline \multirow{2}{*}{ Turbidez (FAU) } & Ultrazym AFP L & $10,15^{\mathrm{a}}$ & $9,43^{\mathrm{a}}$ & $9,68^{\mathrm{a}}$ & $9,52^{\mathrm{a}}$ \\
\hline & Novozym 33095 & $6.500^{\mathrm{b}}$ & $7.175^{\mathrm{b}}$ & $8.508^{\mathrm{a}}$ & $7.258^{\mathrm{a}}$ \\
\cline { 2 - 6 } & Ultrazym AFP L & $8.242^{\mathrm{b}}$ & $10.225^{\mathrm{a}}$ & $8.042^{\mathrm{b}}$ & $8.325^{\mathrm{b}}$ \\
\hline
\end{tabular}

(a) médias seguidas de mesma letra minúscula (a,b,c,d), dentro de cada linha, não diferem entre si a p $\leq 0,05$

Os valores obtidos para o $\mathbf{p H}$, conforme o teste de Tukey, não apresentaram um nível de significância maior que 5\% para as variáveis temperatura, concentração e tempo para as duas enzimas testadas. Assim, as melhores condiçóes foram escolhidas segundo os resultados obtidos para os demais parâmetros avaliados.

Comparando estatisticamente os valores médios da quantidade de sólidos totais nas amostras quanto à variável tempo para as duas enzimas testadas, não foi observada uma diferença significativa $(\mathrm{p}<5 \%)$ para os sólidos totais. No entanto, a variável tempo teve influência nos valores médios dos sólidos totais, gerando diferenças significativas maiores que 5\%. Além disso, apenas a enzima Novozym 33095 apresentou resultados com um nível de significância maior que $5 \%$ para a variável concentração. Do ponto de vista de obter os menores valores de sólidos totais, o tratamento enzimático mais conveniente seria utilizando a enzima Novozym 33095 nas condiçóes de $35^{\circ} \mathrm{C}$, 50 ppm e 15 min.

Outro parâmetro físico-químico cujos valores médios nas amostras foram também estatisticamente comparados foi a Turbidez após o tratamento com as duas enzimas testadas. A análise estatística mostrou que os valores obtidos da turbidez quanto à variação da temperatura não foram significativamente diferentes $(\mathrm{p}<5 \%)$, enquanto que variaçóes nas concentraçóes das enzimas provaram ter significância maior que 5\% nos valores médios da turbidez. Além disso, a variável tempo mostrou significância maior que 5\% nos valores médios da turbidez apenas para o tratamento utilizando a enzima Novozym 33095. Visando a redução da turbidez a valores mínimos, recomenda-se a utilização da enzima Novozym 33095 nas condições de $35^{\circ} \mathrm{C}, 15$ min e concentraçáo de 50 ppm.

Após as diversas condiçóes experimentais testadas para o tratamento enzimático cuja respos- 
ta foi avaliada pela análise dos parâmetros físico-químicos: acidez total, sólidos soluveis ( ${ }^{\circ} \mathrm{Brix}$ ), cor, sólidos totais e turbidez, verifica-se que os melhores resultados, como mostrado na Tabela 7, foram obtidos utilizando a enzima Novozym 33095 na concentraçáo de 50 ppm, temperatura de $35^{\circ} \mathrm{C}$, e tempo de tratamento de $15 \mathrm{~min}$.

No trabalho de Balischi et al. (2002), foi estudado a variaçáo que ocorre na reologia e no diâmetro médio das partículas da polpa integral de acerola submetida a tratamento enzimático, utilizando duas enzimas comerciais: Citrozym Ultra
L e Pectinex Ultra SP-L. Considerando variaçôes nas temperaturas $\left(45\right.$ e $\left.35^{\circ} \mathrm{C}\right)$, nas concentraçóes das enzimas (100 e $120 \mathrm{ppm})$ e nos tempos de tratamento (60 e 75 minutos), alteraçóes nas características reológicas e microscópicas da polpa de acerola foram verificadas após o tratamento, ocorrendo o maior aumento do tamanho das partículas insolúveis c om a enzima Citrozym Ultra - L, e coincidindo com a condiçáo (temperatura de $45^{\circ} \mathrm{C}$, concentraçáo de $100 \mathrm{ppm}$, tempo de tratamento de $60 \mathrm{~min}$ ) que forneceu os melhores resultados para a clarificação.

Tabela 7. Valores dos parâmetros: acidez total, Sólidos solúveis ( ${ }^{\circ}$ Brix), cor, sólidos totais e turbidez obtidos para os sucos de uva comerciais (SC1, SC2 e SC3), polpa de uva in natura e a polpa de uva após o tratamento enzimático com a enzima Novozym 33095, na concentração de $50 \mathrm{ppm}$, temperatura de $35^{\circ} \mathrm{C}$ e tempo de $15 \mathrm{~min}$.

\begin{tabular}{|c|c|c|c|c|c|c|}
\hline Parâmetro & $\begin{array}{c}\text { Polpa de } \\
\text { uva }\end{array}$ & $\begin{array}{c}\text { Polpa após tratamento } \\
\text { com Novozym 33095 }\end{array}$ & SC1 & SC2** & SC3 $^{* *}$ & SCB $^{*}$ \\
\hline Acidez & 13,90 & 12,82 & 9,27 & 11,35 & 10,47 & \\
\hline Sólidos soluveis ( ${ }^{\circ}$ Brix) & 11,0 & 7,4 & 9,0 & 13,5 & 13,5 & $12,8-18,9$ \\
\hline Cor $(\mathrm{mg}$ PCto/L) & 31.550 & 25.900 & 25.400 & 16.800 & 19.600 & \\
\hline $\mathrm{pH}$ & 3,1 & 3,40 & 3,06 & 3,03 & 3,18 & $2,80-3,43$ \\
\hline Sólidos Totais $(\% \mathrm{~m} / \mathrm{m})$ & 7,7 & 6,7 & 13,12 & 12,87 & 12,95 & \\
\hline Turbidez (FAU) & 8.700 & 4.550 & 4.500 & 2.900 & 3.500 & \\
\hline
\end{tabular}

* Rizzon et al. 1998; Rizzon e Miele 1995.

** Sucos de uva comerciais analisados no laboratório.

Considerando as melhores condiçóes dos parâmetros físico-químicas do suco de uva após o tratamento com a enzima Novozym 33095, observa-se uma redução nos valores dos parâmetros físico-químicos em relação à polpa "in natura". Analisando os resultados obtidos para os parâmetros $\mathrm{pH}$ e acidez, verifica-se valores próximos aos obtidos para o sucos comerciais. Entretanto, os parâmetros cor e turbidez tiveram seus valores iniciais reduzidos após o tratamento enzimático, ficando levemente acima dos apresentados para os sucos comerciais. No entanto, esses valores serão ainda mais reduzidos no PSM. Embora os valores dos sólidos solúveis e totais tenham tido uma grande redução ficando muito abaixo dos valores obtidos para os sucos comerciais (SC1, SC2 e SC3) e dos valores médios reportados na literatura (SCB), espera-se que no processo de concentração pelo PSM obter valores iguais ou maiores que os apresentados pelos sucos comerciais. A re- dução nos valores dos parâmetros físico-químicos é essencial como etapa preliminar aos PSM para preservar a eficiência da membrana, minimizando a formação de camada de torta e a polarização por concentração. Desta forma, o pré-tratamento enzimático da polpa de uva se mostrou uma etapa necessária e eficiente no processo de fabricaçáo do suco de uva.

\section{CONCLUSÃO}

O pré-tratamentos enzimático, na fabricação do suco de uva, se mostrou um processo eficiente na redução dos valores dos parâmetros físico-químicos: acidez total, sólidos solúveis ( ${ }^{\circ}$ Brix), cor, sólidos totais e turbidez. Reduzindo assim as partículas insolúveis e materiais em suspensão, e com isto deixando a polpa com as condiçóes necessárias para o próximo processo na fabricação do suco de uva (PSM). 


\section{AGRADECIMENTOS}

À Fundação Araucária pelo financiamento do Projeto.

\section{REFERÊNCIAS}

AOAC (Association of Official Analytical Chemists). Official methods of analyses of A. O. A. C. $15^{\text {th }}$ ed. Washington: AOAC, 1990. v. 2, cap. 37, p. 915 e 922 .

BALISCHI, L, PEREIRA, N. C., LIMA, O. C. DA M., BARROS, S. T. D. DE, DAMASCENO, L. W., MENDES, E. S. Influência do tratamento enzimático sobre as características reológicas e microscópicas da polpa de acerola. Acta Scientiarum. Technology, Vol. 24 (2002)

BALISCHI, L. Tratamento enzimático da polpa integral de acerola empregando as enzimas pectinex Ultra SP-L e Citrozym Ultra L. Maringá, PR, 2002. Originalmente apresentada como dissertação de mestrado, Universidade Estadual de Maringá, Departamento de Engenharia Química. 87p.

BARROS, S.T.D. Clarificação dos sucos de acerola e abacaxi por ultrafiltração: Modelagem e Simulação do Fluxo de Permeado e Determinação dos Mecanismos de Fouling, 2002. Tese (Doutorado) - DTP/Unicamp, Campinas, 2002.

CASSANO, A., DRIOLI, E., GALAVERNA, G., MARCHELLI, R., DI SILVESTRO, G., CAGNASSO, P., Clarification and concentration of citrus and carrot juices by integrated membrane processes, Journal of Food Engineering, v. 57 , p.153-163, 2003.

CHERYAN, M., ALVAREZ, J. R. Food and beverage industry aplications. Departament of Food Science, 103 Agricultural Bioprocess Laboratory, University of Lilinois, Urbana, IL 61801, USA.
Departament of Chemical Engineering, University of Oviedo, Spain. 1995.

HABERT, A. C.; BORGES, C. P. e NÓBREGA, R., Processos de Separação por Membranas, Rio de Janeiro: E-papers, 2006, 180 p.

INSTITUTO ADOLFO LUTZ. Normas analíticas do Instituto Adolfo Lutz: Métodos químicos e físicos para análises de alimentos. 3.ed. Sáo Paulo, 1985. v. 1, p. 27.

MARZAROTTO, V., Suco de Uva. In: Waldemar Gastoni Venturini Filho. (Coord.) Tecnologia de bebidas: matéria-prima, processamento, $\mathrm{BPF} /$ APPCC, legislação e mercado. São Paulo: Edgard Blücher, 2005, p.311-345.

NOVOZYMES Manual, Product Sheet, Fruit \& Vegetable-07212-02. Araucária: Novozymes Latin America Limited. 2001.

PETRUS, J.C.C. Preparação, modificação e caracterizaçáo de membrana assimétrica para clarificação de suco de frutas. 1997. Tese (Doutorado) - FEA/Unicamp, Campinas, 1997.

RIZZON, L. A., MANFROI, V., MENEGUZZO, J. Elaboração de suco de uva na propriedade vitícola. Bento Gonçalves: EMBRAPA Uva e Vinho, 1998. 24p. (Documentos, 21).

RIZZON, L. A., MIELE, A. Características analíticas de sucos de uva elaborados no Rio Grande do Sul. Boletim Sociedade Brasileira de Ciências e Tecnologia de Alimentos, Campinas, v. 29, n.2, p. 129-133, jul./dez. 1995.

YAMAGUCHI, T., NIITSUMA, T., BALAGOPAL, N. N., KAZUAKI, N., Lithium silicate based membranes for high temperature $\mathrm{CO} 2$ separation, Journal of Membrane Science 294 (2007) 16-21. 